\title{
Fibrocytes, Inflammation, and Fibrosis in Crohn's Disease: Another Piece of the Puzzle
}

\author{
D. Sorrentino
}

Published online: 11 October 2013

(C) Springer Science+Business Media New York 2013

In the gut and in other organs, tissue repair following damage results from the complex interplay of chemokines, cytokines and growth factors that recruits and activates cells into the site of injury. During the initial inflammatory phase, monocytes and neutrophils remove the damaged tissue, secreting substances that activate acquired immunity, promoting tissue repair. Fibroblast activation and recruitment (as well as angiogenesis) occur at this time, mostly in response to increasing concentrations of growth factors and proinflammatory cytokines such as IL-1, IL-6, and TNF- $\alpha$ [1]. Subsequently, re-epithelialization accompanied by collagen/extracellular matrix (ECM) deposition and remodeling take place until specialized cells with contractile capability such as fibroblast-derived myofibroblasts restore original tissue integrity [2].

During chronic inflammation, such as that associated with inflammatory bowel diseases (IBD), excessive accumulation of ECM might lead to tissue fibrosis. While ECM deposition in ulcerative colitis appears to be limited to the superficial layers of bowel wall, in Crohn's disease (CD) it often involves the entire wall thickness and may produce strictures, a major morbid complication of $\mathrm{CD}$, often requiring surgical intervention [3]. While anti-inflammatory therapies are commonplace, little progress has been made to develop effective therapies to prevent or reverse intestinal fibrosis in CD.

D. Sorrentino $(\bowtie)$

Division of Gastroenterology, IBD Center, Virginia TechCarilion School of Medicine, 3 Riverside Circle, Roanoke, VA 24016, USA

e-mail: drsorrentino@ carilionclinic.org

D. Sorrentino

Department of Clinical and Experimental Medical Sciences, University of Udine Medical School, Udine, Italy
Although fibrosis results primarily from the rapid proliferation of the resident (local) fibroblast population in response to inflammation [4], several other mechanisms appear to account for fibrosis during inflammation, which include fibroblast migration from the surrounding tissue layers, migration and differentiation of stellate cells, epithelial- and endothelial-to-mesenchymal transition (a process by which endothelial and epithelial cells lose their typical markers and acquire a spindle shape morphology), pericyte differentiation (whereas capillary lining cells assume the morphology and functions of fibroblasts) and extraintestinal fibroblast recruitment [1]. The latter might involve migration from the bone marrow and further differentiation into the site of inflammation of either mesenchymal stem cells (typical stromal cells, fibroblast-like) or hematopoietic stem cells-also called fibrocytes.

While the relevance of many of these mechanisms in fibrosis development is well established for several organs, much remains to be discovered in the process of intestinal fibrosis. This is particularly true for fibrocytes, a cell line belonging to circulating "inflammatory monocytes" that normally replenish tissue macrophages and dendritic cells. During inflammation, under the influence of TGF- $\beta$ and upon contact with T-cells, these monocytes downregulate their hematopoietic markers-CD14, CD34 and CD45and upregulate collagen synthesizing enzymes, becoming circulating tissue fibrocytes and myofibroblasts (reviewed in [5]). Though their involvement in fibrosis development in the skin, lung, pancreas, kidney, neoplasias and blood vessels is reasonably well documented [5], their participation in the development of intestinal fibrosis is currently poorly understood.

Sazuka et al. [6], in the current issue of Digestive Diseases and Sciences, implicate fibrocytes for the first time in the development of fibrosis in CD. Their report follows 
another study from the same group in which-in a murine model of colitis - the authors demonstrated that fibrocytes are recruited in the inflammatory phase of colitis and differentiate into spindle-shaped cells in the healing phase [7]. In their study, the authors first show that in surgically resected specimens of patients with $\mathrm{CD}$, the ratio of fibrocytes to total leukocytes is greatly increased only in inflamed areas whereas in non-inflamed areas such ratio is similar to that in the peripheral blood. In established fibrotic lesions, the relative number of fibrocytes was similar to that of non-inflamed areas, likely indicating that fibrocytes are recruited early during the inflammatory process and then differentiate into typical fibroblasts/ myofibroblasts during fibrosis development. Secondly, Sazuka et al. report that the ratio of circulating fibrocytes relative to total circulating leukocytes is significantly increased in CD patients compared to healthy controlsand that these cells appear to be "set" to be recruited in sites of inflammation. Finally, cultured fibrocytes isolated from $\mathrm{CD}$ patients and healthy controls highly upregulate tumor necrosis factor- $\alpha$ (TNF- $\alpha$ ) and collagen type-I (ColI) mRNA expression in response to lipopolysaccharide (LPS), a bacterial component with well-established pro-inflammatory properties due to activation of the pattern recognition molecule Toll-like receptor 4 (TLR4). TNF- $\alpha$ appears not only to mediate inflammation but also to increase fibroblast retention at the inflammatory site-thus contributing to the development of fibrosis [8].

Collectively, these data indicate that fibrocytes are likely major contributors to the development and persistence of inflammation and fibrosis in CD. Unfortunately, the authors do not provide details on the genetic background, disease duration, concomitant therapy and disease location (i.e. ileal vs colonic) in studied patients, information which could add to our knowledge of how fibrocytes are involved in CD pathogenesis. Moreover, as the authors point out, they took "still photograph" of many sequential events; fibrocyte tagging is necessary to make a "motion picture" in order to fully understand and verify the complete dynamics of the process.

Understanding the mechanisms of disease is the obvious first step in the development of novel therapies-more so for the lagging field of fibrosis in CD. Based on current knowledge, including the observations by Sazuka et al., early and vigorous suppression of inflammation could halt disease evolution and hence prevent fibrosis development. Nevertheless, CD patients usually seek medical attention when the disease has become symptomatic - not at its biological onset. Likely, by then, the fibrotic mechanisms - activated by inflammation - might have become independent and irreversible. Moreover, a proportion of patients may develop fibrosis and strictures regardless of early suppression of inflammation due to genetic factors
[9], a hypothesis supported by the finding that the incidence of surgery in $\mathrm{CD}$ (typically performed for fibrotic complications) does not appear to have declined in the era of the powerful anti-TNF agents [10]. The latter observation however may be affected by the limited and often late use of biological agents in the early years of their introduction. In support of this supposition, reduction of surgery rates is reported in studies in which anti-TNF therapy was started early in the disease course (reviewed in [3]).

Targeting fibrocytes to block or reverse fibrosis, as suggested by Sazuka et al. [6], appears particularly attractive since these cells might not only contribute towards fibrosis development but also amplify inflammation-as exemplified by the secretion of large amounts of TNF- $\alpha$ in response to LPS stimulation. Anti-TNF antibodies induce apoptosis in myofibroblasts [11]; whether they are also capable of inducing fibrocyte apoptosis-and hence block inflammation and fibrosis as speculated by Sazuka et al.-is unclear at this time.

As of today, no therapy seems to be capable of truly reversing fibrosis in $\mathrm{CD}$ [1], although specific molecules are being studied for the purpose [12]. For the time being, this and other studies [13] do reinforce the concept that early, aggressive suppression of inflammation with the most powerful agents is still the best strategy to avoid subsequent stricture formation in $\mathrm{CD}$.

\section{References}

1. Rieder F, Fiocchi C. Intestinal fibrosis in inflammatory bowel disease-current knowledge and future perspectives. J Crohn's Colitis. 2008;2:279-290.

2. Quan TE, Cowper SE, Bucala R. The role of circulating fibrocytes in fibrosis. Curr Rheumatol Rep. 2006;8:145-150.

3. Sorrentino D, Fogel S, van den Bogaerde J. Surgery for Crohn's disease and anti-TNF agents. The changing scenario. Exp Rev Gastroenterol Hepatol. 2013. doi:10.1586/17474124.2013.842895.

4. Lawrance IC, Maxwell L, Doe W. Altered response of intestinal mucosal fibroblasts to profibrogenic cytokines in inflammatory bowel disease. Inflamm Bowel Dis. 2001;7:226-236.

5. Mifflin RC, Pinchuk IV, Saada JI, Powell DW. Intestinal myofibroblasts: targets for stem cell therapy. Am J Physiol Gastrointest Liver Physiol. 2011;300:G684-G696.

6. Sazuka S, Katsuno T, Nagakawa T, et al. Fibrocytes are involved in inflammation as well as fibrosis in the pathogenesis of Crohn's disease. Dig Dis Sci. 2013 (Epub ahead of print). doi:10.1007/ s10620-013-2813-8.

7. Uehara H, Nakagawa T, Katsuno T, et al. Emergence of fibrocytes showing morphological changes in the inflamed colonic mucosa. Dig Dis Sci. 2010;55:253-260.

8. Leeb SN, Vogl D, Gunckel M, et al. Reduced migration of fibroblasts in inflammatory bowel disease: role of inflammatory mediators and focal adhesion kinase. Gastroenterology. 2003;125:1341-1354.

9. Wynn TA. Fibrotic disease and the $\mathrm{T}(\mathrm{H}) 1 / \mathrm{T}(\mathrm{H}) 2$ paradigm. Nat Rev Immunol. 2004;4:583-594.

10. Jones DW, Finlayson SR. Trends in surgery for Crohn's disease in the era of infliximab. Ann Surg. 2010;252:307-312. 
11. Di Sabatino A, Pender SL, Jackson CL, et al. Functional modulation of Crohn's disease myofibroblasts by anti-tumor necrosis factor antibodies. Gastroenterology. 2007;133:137-149.

12. Oshitani N, Yamagami H, Watanabe K, Higuchi K, Arakawa T. Long-term prospective pilot study with tranilast for the prevention of stricture progression in patients with Crohn's disease. Gut. 2007;56:599-600.
13. Johnson LA, Luke A, Sauder K, Moons DS, Horowitz JC, Higgins PD. Intestinal fibrosis is reduced by early elimination of inflammation in a mouse model of IBD: impact of a "top-down" approach to intestinal fibrosis in mice. Inflamm Bowel Dis. 2012;18:460-471. 